\title{
Is a postintubation chest radiograph necessary in the emergency department?
}

\author{
Daniel C. McGillicuddy • Matthew R. Babineau • \\ Jonathan Fisher • Kevin Ban • Leon D. Sanchez
}

Received: 16 June 2009 / Accepted: 29 September 2009/Published online: 19 November 2009

(C) Springer-Verlag London Ltd 2009

\begin{abstract}
Background Postintubation chest X-rays (CXR) are standard practice in emergency department (ED) intubations. In the operating room, it is not usually a standard practice to confirm endotracheal tube placement with a CXR.

Aims We seek to study the utility of postintubation CXR in ED patients.

Methods This was a retrospective case series of 157 adult patients intubated in the ED of an urban academic hospital with an emergency medicine training program. Standardized chart review was performed by two emergency physicians (EP) using a structured data abstraction tool and final radiology attending reads of postintubation CXR to assess placement. Endotracheal tube placement was graded as satisfactory, too high, too low, or malpositioned in the esophagus. Descriptive statistics were used, and $95 \%$ confidence intervals (CI) were reported. Hospital Institutional Review Board approval was obtained.

Results A total of 157 patients were intubated in the ED during the study period: 127 (81\%, 95\% CI: 74-86) had adequate tube placement by CXR confirmation, $9(6 \%$, 95\% CI: 3-11) endotracheal tubes were judged to be too high, and $20(13 \%, 95 \% \mathrm{CI}: 8-19)$ were judged to be too low with $10(6.5 \%, 95 \% \mathrm{CI}: 3-11)$ of these being right mainstem bronchus intubations. One patient $(<1 \%$, 95\% CI: $<0.0001-4)$ had a CXR confirming esophageal intubation.
\end{abstract}

D. C. McGillicuddy $\cdot$ M. R. Babineau $\cdot$ J. Fisher $\cdot$ K. Ban $\cdot$

L. D. Sanchez $(\bowtie)$

Department of Emergency Medicine,

Beth Israel Deaconess Medical Center,

One Deaconess Road, West Campus Clinical Center, 2nd Floor,

Boston, MA 02215, USA

e-mail: Lsanche1@bidmc.harvard.edu
Conclusion ED intubations were judged to have "satisfactory" placement by CXR in $81 \%$ of patients. CXR is able to identify a small subset of patients that likely need immediate intervention based on their CXR. Until further studies refute the utility of postintubation CXR in ED intubations, they should remain a part of routine practice.

Keywords Intubation · Chest X-ray - Emergency department

\section{Introduction}

Airway management skills are essential for the emergency physician (EP). Several studies have compared EPs with others, primarily anesthesiologists, to demonstrate that EPs are as good as other groups that routinely intubate [1,2]. Standard practice in US emergency departments (ED) is to obtain a postintubation chest X-ray (CXR) to confirm endotracheal (ET) tube placement. In other hospital settings, particularly the operating room, postintubation CXRs are not routine. In a survey of EDs in the Tuscan region of Italy, variation in the practice of obtaining a postintubation CXR was found, with $50.7 \%$ of providers routinely obtaining one [3]. The objective of this study was to determine the proportion of ED intubations that are malpositioned on CXR and to examine whether the standard US practice of a postintubation CXR is necessary.

\section{Materials and methods}

A retrospective chart review was undertaken for all ED intubations performed between 1 January 2004 and 31 December 2004 at an urban tertiary care center with approximately 50,000 ED visits. Standardized chart review 
was performed by two EPs using a structured data abstraction tool and final radiology attending reads of postintubation CXRs to assess the utility of CXRs on postintubated patients.

The analysis included 157 patients who were obtained by examining the hospital database for all ED patients during that time period for which an intubation procedure was recorded. Our institution does not see pediatric patients so these were not included. Patients intubated prior to arrival at our hospital were not included in the analysis.

The official radiology report of the postintubation CXR was obtained for all patients. Information on endotracheal tube placement was obtained from the medical record. The endotracheal tube position was graded as satisfactory, too high, too low, or malpositioned in the esophagus. The tube location was assessed by an attending radiologist.

Data were entered into a Microsoft Excel 2003 (Redmond, WA, USA) database. The study was approved by the hospital Institutional Review Board. Descriptive statistics were used and $95 \%$ CI were reported as appropriate. Graphpad 2002 (San Diego, CA, USA) software was used in data analysis.

\section{Results}

A total of 157 patients were intubated in the ED during the study period. Prehospital intubations and transferred intubated patients were excluded. Of the patients included in the study, $127(81 \%, 95 \%$ CI: 74-86) had adequate tube placement by CXR confirmation. Nine (6\%, 95\% CI: 3-11) endotracheal tubes were judged to be too high and required advancement. Twenty (13\%, 95\% CI: 8-19) endotracheal tubes were judged to be too low, and ten $(6.5 \%, 95 \% \mathrm{CI}$ : 3-11) of these low endotracheal tubes were right mainstem bronchus intubations. Of the right mainstem intubations nine of ten $(90 \%, 95 \%$ CI: $57-99)$ were in women. One patient $(<1 \%, 95 \% \mathrm{CI}:<0.0001-4)$ had a CXR confirming esophageal intubation.

\section{Discussion}

Visualization of the ET tube as it passes the vocal cords, auscultation for bilateral breath sounds, and colorimetric $\mathrm{CO}_{2}$ detectors are routinely used as primary methods of confirmation of ET tube placement. Postintubation CXRs are not regarded as a primary method of confirmation. Even in the ED, use of postintubation CXR is not universal $[3,4]$. Prior studies give us some information on the rate of inadequate tube positioning after intubation.
As in previous studies by Schwartz et al. and Brunel et al., we found that women are at greater risk for malpositioning of the tube too close to the carina $[5,6]$. Nine of the ten mainstem intubations identified by radiograph in our study were in women. Our rate of mainstem intubation of $6.5 \%$ is similar to the rate in the Brunel et al. study of $5 \%$ [6]. By contrast eight of nine ET tubes judged to be too high by the radiologist were in men. Although Roberts et al. advocate the insertion of the ET tube to a different prespecified depth for men and women to reduce the rate of malpositioning, Schwartz et al. and Brunel et al. both do not find the practice reliable enough to obviate the need for CXR [5-7]. The rate of ET tube malpositioning is between 9.6 and $15.5 \%$ in previous studies [5-7]. Our rate of $19.1 \%$ is higher than those other studies: however, we are relying on the radiologist's opinion.

Some of the CXRs read as too high or too low may not have been read as such by the EP. Furthermore, in patients with difficult airways, the EP may not want to adjust an ET tube that is deemed too deep by breath sounds until a confirmatory CXR has been obtained, due to the theoretical chance of tube dislodgement in a difficult airway. Even though the use of $\mathrm{CO}_{2}$ colorimetric devices and postintubation auscultation are routine in our ED, the CXR identified ten mainstem intubations and one esophageal intubation that went unrecognized prior to the CXR.

\section{Limitations}

As a single-center retrospective study we were limited in our ability to make general statements based on our study population. As we saw no pediatric patients we can make no statements about this patient population. We are also limited by the information included in the radiology report. It is possible that the chart review missed some ED intubations if they were not documented. If this happened we cannot say how inclusion of those intubations would affect our numbers. Finally, as we deemed the final radiology report of ET tube position as the reference standard, some may argue that the EP's preliminary read of ET tube position had validity as well.

We did not correlate the ED read from the chart as ED documentation of the ED read was often missing or incomplete. Documentation as to tube adjustment in the chart is very limited and we could often not tell from the chart when or whether it was done. We cannot tell based on the chart review how quickly these findings were acted on. It is unlikely that ED doctors waited to adjust ET tubes until the radiology read became available, but we cannot make any statements about this based on this retrospective study. 


\section{Conclusions}

Based on our results we support the use of postintubation CXR as a method to corroborate ET tube placement after primary confirmation methods have been used. In our study population the CXR identified malpositioning in $19 \%$ of the patients. By the most conservative estimate the CXR was instrumental in $7 \%$ of cases in identifying mainstem or esophageal intubations that went unrecognized by the use of other methods to confirm placement. Several of the malpositioned ET tubes likely needed emergent adjustment, including removing from the right mainstem, and removal from the esophagus and subsequent tracheal intubation. As the CXR is an inexpensive test that is noninvasive and can provide data on ET tube malposition in ED intubations, it is our feeling that a postintubation CXR in the ED should remain a standard of care. In our department ED physicians routinely review postintubation films without waiting for a radiology read, and routine review of postintubation films would lead to recognition of ET tube malpositioning.

\section{References}

1. Levitan RM, Rosenblatt B, Meiner EM et al (2004) Alternating day emergency medicine and anesthesia resident responsibility for management of the trauma airway: a study of laryngoscopy performance and intubation success. Ann Emerg Med 43(1):48-53

2. Bushra JS, McNeil B, Wald DA et al (2004) A comparison of trauma intubations managed by anesthesiologists and emergency physicians. Acad Emerg Med 11(1):66-70

3. Sanchez LD, Di Martino P, Babineau M, Lanigra M, Ban KM (2008) Intubation practice patterns in Tuscan emergency departments. Int J Emerg Med 1:127-129

4. Easley RB, Segeleon JE, Haun SE et al (2000) Prospective study of airway management of children requiring endotracheal intubation before admission to a pediatric intensive care unit. Crit Care Med 28(6):2058-2063

5. Schwartz DE, Lieberman JA, Cohen NH (1994) Women are at greater risk than men for malpositioning of the endotracheal tube after emergent intubation. Crit Care Med 22(7):1127-1131

6. Brunel W, Coleman DL, Schwartz DE, Peper E, Cohen NH (1989) Assessment of routine chest roentgenograms and the physical examination to confirm endotracheal tube position. Chest 96(5):1043-1045

7. Roberts JR, Spadafora M, Cone DC (1995) Proper depth of placement of oral endotracheal tubes in adults prior to radiographic confirmation. Acad Emerg Med 2(1):20-24 